\title{
Smoking cessation
}

\section{Stephen A Margolis}

Now that I'm gone, I tell you: don't smoke, whatever you do. Just don't smoke.

- Yul Brynner ${ }^{1}$

Tobacco has been a pervasive part of the human experience since prehistory, with tobacco cultivation in the Americas dating back to $6000 \mathrm{BC} .^{2}$ The dangers of tobacco smoking were clearly apparent and publicised by the mid-20th century, but formal measures to stop smoking were slow to be introduced or accepted. Looking back from our current understanding, it is hard to believe that around the time when The Royal Australian College of General Practitioners (RACGP) was established, doctors including general practitioners (GPs) were promoting smoking to patients, ${ }^{3}$ with brand preference among doctors used as a marketing strategy. ${ }^{4}$ Even a noted GP and past president of the RACGP 'smoked Temple Bar cigarettes, sometimes while consulting'. ${ }^{5}$

The falling rate of cigarette smoking is a remarkable Australian public health success story, with the age-standardised daily smoking rates falling from $25.6 \%$ (1989-90) to $14.7 \%(2014-15){ }^{6}$

However, the smoking rate among Aboriginal and Torres Strait Islander peoples remains unacceptably high despite decreasing from 50\% (2004-05) to $40 \%$ (2018-19). ${ }^{7}$

Health authorities and governments require eternal vigilance as the threat of harm from tobacco products remains. For example, although direct advertising of tobacco products has been prohibited in Australia for decades, film and television often use pro-smoking imagery and glamorisation. ${ }^{8}$ The World Health Organization (WHO) is a strong supporter of the public health approach model, noting that surveillance is the key to guiding effective intervention policies. ${ }^{9}$

In addition to strategies to prevent people from starting to smoke, there has also been an upsurge of evidencebased medical strategies to help those who are smoking to stop. Success in stopping requires specific supports and intervention to improve the $4 \%$ unsupported success rate. ${ }^{9}$

In Australia, the RACGP has been at the forefront of supporting health staff in this endeavour, including through the publication of formal guidelines. ${ }^{10}$ The WHO recently awarded the RACGP's work in this area at the World No Tobacco Day 2020 awards. ${ }^{11}$

\section{Australian Journal of General Practice} $(A J G P)$ is fortunate to have Professor Nick Zwar, the Chair of the RACGP Expert Advisory Group on smoking cessation, writing for AJGP this month. ${ }^{12}$ In his article, Professor Zwar summarises and highlights the key clinical issues that guide evidence-based clinical practice in this area. It is becoming clear that a combination of behavioural and pharmacological treatments is the most effective method. Effective behavioural techniques for clinicians to encourage cessation that are explored in this article focus on the brief intervention approach ('Ask, Advise, Help'). Additionally, the latest approaches in pharmacotherapy are considered, including the emerging evidence that combination nicotine replacement therapy (patch and short-acting oral form) or varenicline are the most effective. The article also discusses the controversies relating to nicotine-containing e-cigarettes.

Tobacco use remains a global health issue, with core components including both discouraging people from starting and helping those who want to stop. As unsupported stopping is rarely successful, effective evidence-based smoking cessation programs continue to be a core component of the public health response to smoking.

\section{Author}

Stephen A Margolis, OAM; Editor in Chief, Australian Journal of General Practice; Professor, School of Medicine, Griffith University, Qld; Staff Specialist, Royal Flying Doctor Service

\section{References}

1. United Press International. Yul Brynner's posthumous message. Los Angeles Times. 20 February 1986.

2. World Health Organization. The history of tobacco. Geneva, Switzerland: WHO, [date unknown] Available at www.who.int/tobacco/en/atlas2.pdf [Accessed 1 July 2020].

3. Elliot S. When doctors, and even Santa, endorsed tobacco. The New York Times. 6 October 2008.

4. Starts at 60 writers. Lighting up in the '50s: When smoking was cheap and trendy. Starts at 60. 2 June 2019.

5. Kamien M. Dr James Richard Henry Watson CMG An RACGP past president. The Royal Australian College of General Practitioners Archives Committee, [date unknown]. Available at www. racgp.org.au/the-racgp/history/biographies/ dr-james-watson-cmg [Accessed 1 July 2020].

6. Australian Institute of Health and Welfare Australia's health 2018. Cat. no. AUS 221. Canberra, ACT: AlHW.

7. Colonna E, Maddox R, Cohen R, et al. Review of tobacco use among Aboriginal and Torres Strait Islander peoples. Perth, WA: Australian Indigenous HealthInfoNet, 2020.

8. Wood L, Letcher T, Winstanley M. 5.16 Smoking in movies, TV and other popular culture/media. In: Greenhalgh EM, Scollo MM, Winstanley MH, editors. Tobacco in Australia: Facts and issues. Melbourne, Vic: Cancer Council Victoria, 212. Available at www.tobaccoinaustralia.org.au/ chapter-5-uptake/5-16-smoking-in-movies-tvand-other-pop [Accessed 2 July 2020].

9. World Health Organization. Tobacco. Geneva, Switzerland: WHO, 2020. Available at www. who.int/news-room/fact-sheets/detail/tobacco [Accessed 2 July 2020].

10. The Royal Australian College of General Practitioners. Supporting smoking cessation: A guide for health professionals. 2nd edn. East Melbourne, Vic: RACGP, 2019.

11. World Health Organization. World No Tobacco Day 2020 awards - The winners. Geneva, Switzerland: WHO, 2020. Available at www.who. int/news-room/detail/22-05-2020-world-notobacco-day-2020-awards-the-winners [Accessed 2 July 2020].

12. Zwar N. Smoking cessation. Aust J Gen Pract 2020;49(8):474-81. 